\title{
Trayectorias personales de lectura: autobiografías lectoras de estudiantes de pedagogía en una universidad chilena'
} \author{
Students from a Chilean University \\ CAROLINA MERINO \\ SOLEDAD BARRERA \\ GABRIELA ANDREA ALBORNOZ \\ Universidad Católica del Maule \\ Chile \\ cmerino@ucm.cl \\ sbarrera@ucm.cl \\ gabriela.albornoz@alu.ucm.cl
}

Personal Reading Trajectories: Reading Autobiographies of Pedagogy

(Recibido: 25-OI-2OI9; aceptado: $24^{-\mathrm{OI}-2 \mathrm{O} 2 \mathrm{O})}$

Resumen. En los últimos años, han surgido estudios sobre las prácticas lectoras del profesorado, a partir del supuesto de que la futura mediación lectora se relaciona con la propia trayectoria en este ámbito. En este contexto, se presenta una investigación cualitativa que analiza 279 autobiografías lectoras escritas por ocho generaciones de estudiantes chilenos de pedagogía, a lo largo de la última década. Se trata de un estudio exploratorio y diacrónico que empleó la técnica "historias de vida" para recolectar información acerca de las trayectorias personales y formas de apropiación de la lectura. Los datos se abordaron a través de un análisis mixto (estadístico y de contenido) que estableció categorías estructurales y subcategorías relacionadas con las experiencias lectoras en educación inicial, primaria, secundaria y universitaria; los momentos y lugares donde se realiza esa lectura; el rol de los mediadores y el valor atribuido a la literatura. Los principales resultados apuntan a destacar la centralidad de la lectura compartida, el rol mediador de las figuras familiares y la escasa participación de la escuela y la biblioteca en esta tarea. La razón radicaría en la obligatoriedad con que se aborda la lectura en el espacio escolar, alejándola del placer y del goce estético.

Palabras clave: formación inicial docente; prácticas lectoras, autobiografias; familia; escuela.
Abstract. In recent years, studies about teachers' reading practices have emerged, based on the assumption that future reading mediation is related to their own trajectory in this field. In this context, a qualitative research was developed that analyzes 279 reading autobiographies written by eight generations of Chilean pedagogy students, throughout the last decade. This exploratory and diachronic study worked with the "life stories" technique to collect information about the personal trajectories and forms of reading appropriation. The data was addressed by means of mixed analysis (statistical and of content) that established structural categories and subcategories related to the reading experiences in pre-school, primary, secondary and university education; the moments and places where the reading takes place; the mediators role and the value attributed to literature. The main results highlight the centrality of shared reading, the family members acting as mediators and the limited participation of the school and the library in this task. The reason would lay in the compulsory nature of reading in the school context, taking it away from pleasure and aesthetic enjoyment.

Keywords: initial teacher training; reading practices; autobiographies; family; school.

\footnotetext{
${ }^{\text {I }}$ Para citar este artículo: Merino, Carolina; Barrera, Soledad. y Albornoz, Gabriela A. (2O2O). Trayectorias personales de lectura: autobiografías lectoras de estudiantes de pedagogía en una universidad chilena. Alabe 22 . [www.revistaalabe.com] 


\section{Introducción}

La formación de lectores, particularmente de literatura, constituye en la actualidad una preocupación para los distintos países que diseñan y ponen en marcha ambiciosos planes y costosas campañas promocionales sin obtener los resultados esperados.

En el caso de los niños y jóvenes, esta falta de resultados se relaciona, tal vez como razón principal, con las posibilidades que ofrecen las nuevas tecnologías de la comunicación: "La era audiovisual, primero, y digital, después, han revolucionado las expectativas de formación y de ocio de niños y adolescentes” (Dueñas, Tabernero, Calvo y Consejo, 2OI4: 22).

Tal como plantean algunos autores, la digitalización del conocimiento y su intercambio se han extendido al punto de hablarse de Escuela 2.0, entendiendo por ello una red en que los internautas son al mismo tiempo consumidores y productores de contenidos (Cassany,2OI2; Leibrandt,2OIO).

Como anticiparon Cerrillo y Senís (2005), se ha ido configurando un modelo diferente de lector, condicionado por estos nuevos modos de acceso a la información. Ellos modifican la relación con los textos literarios, con el libro como soporte dominante y establecen nuevos hábitos y prácticas lectoras.

Lluch (2OI3) plantea que los espacios virtuales como redes sociales, blogs, foros, entre otros, se conciben como un fenómeno de comunicación social, pero especialmente como una herramienta para la promoción de la lectura entre los más jóvenes, quienes se congregan "para hablar de libros, lecturas y autores" (Lluch, Citado en Prats y Contreras, 2OI3: 4I).

La didáctica de la literatura no puede desaprovechar las posibilidades que ofrecen estas nuevas rutas de acceso a la lectura literaria. Prats y Contreras (20I3) sugieren, a partir de su experiencia como docentes en la formación inicial de profesores, nuevas propuestas de actuación ancladas en la multimodalidad y la conectividad como elementos centrales. Entre ellas mencionan las siguientes: herramientas digitales para fomentar la lectura en el aula basadas en la valoración y recomendación de lecturas, plataformas para desarrollar la creatividad literaria y recursos como el booktrailer para presentar los libros.

Respecto de este último, Tabernero (20I3) afirma que "se convierte en un instrumento no solo de promoción de la lectura sino también de desarrollo de la competencia literaria en el marco de la escuela 2.o que entiende la construcción del conocimiento de una forma interactiva y compartida" (p.2I9).

Es en este particular escenario, donde los futuros profesores comenzarán su labor como educadores de las nuevas generaciones, a quienes habrá que ofrecer una educación lectora y literaria de calidad.

En el campo de la didáctica de la literatura existen diversas líneas de estudio, entre las cuales se encuentran las prácticas lectoras del profesorado y de quienes se preparan para ello, y su posible influencia en la mediación lectora en el ámbito escolar (Munita, 2OI3). 
Es así como desde fines de los años 9o, se observa una motivación creciente por estudiar las biografías lectoras de los futuros docentes, a partir de interrogantes que los conectan con su trayectoria pasada y presente, y con su autopercepción como lectores y próximamente profesores (Colomer y Munita, 2OI3; Munita, 2OI6; Dueñas et al., 2OI4).

Granado y Puig (2OI4) proponen cuatro propósitos de indagación que se solapan y complementan. El último de ellos apunta a:

Utilizar las narrativas de historias de lectura como estrategia de formación de los docentes, es decir, como herramienta metodológica para ayudarles a mejorar la comprensión de sí mismos, modificar sus creencias, tomar conciencia de sus conocimientos previos sobre lectura, comprender la diversidad de trayectorias lectoras, abrirse a nuevas ideas, etc. Estos estudios suelen analizar el impacto de esta estrategia en su capacitación docente (p. 44).

En la misma línea, Munita (2OI7) postula que el interés por los hábitos lectores del profesorado ha impulsado una serie de estudios que exploran las prácticas culturales del mediador en relación a la lectura. En el ámbito hispanohablante ha sido abordado desde disciplinas tan diversas como la sociología de la lectura o la didáctica de la lengua y la literatura:

Desde una perspectiva didáctica, estudiar al profesor como lector en su espacio personal se ha sustentado en una idea clave: la educación literaria necesita de mediadores de lectura capaces de transmitir su propia relación personal (y pasional) con el libro y la lectura (p.2).

Dichas conclusiones se derivan de experiencias realizadas en España con docentes en ejercicio y estudiantes de magisterio de educación infantil y educación primaria, con la finalidad de proyectar acciones de formación docente, como diseñar un itinerario lector para el mediador.

En Chile, se cuenta con una Política del Libro y un Plan Nacional de la Lectura (2OI5-2O2O) que busca lograr la mayor cobertura territorial posible consolidando planes regionales. Una de sus líneas estratégicas son los Estudios, que considera entre sus objetivos:

[...] la generación y difusión de conocimiento (investigaciones, seminarios, encuestas, etc.) que aporten a la toma de decisiones y definiciones sobre las materias que conciernen al campo de la lectura como, por ejemplo, hábitos y comportamiento lector, uso de bibliotecas, rol de los mediadores, nuevos formatos y soportes, entre otras (Consejo Nacional de la Cultura y las Artes,20I5: 47).

No obstante lo anterior, hasta la fecha no existen investigaciones similares a las 
realizadas en contexto español, cuyos resultados puedan contribuir a repensar los procesos de formación docente en educación literaria.

En nuestro estudio, intentaremos aportar a la generación de conocimiento sobre este tema, al centrarnos en el análisis de 279 autobiografías lectoras escritas por ocho generaciones de estudiantes chilenos de pedagogía, a lo largo de la última década.

\section{Método}

Partimos del siguiente supuesto de investigación, haciendo nuestro el planteamiento de Munita (2OI3): la posibilidad de éxito de la futura mediación de los profesores se relaciona con su propia trayectoria lectora.

La indagación se realizó con una orientación cualitativa, bajo un enfoque hermenéutico-interpretativo y desde el método biográfico-narrativo. Se trata de un estudio exploratorio y diacrónico que empleó la técnica "historias de vida” para recolectar la información acerca de las trayectorias personales y formas de apropiación de la lectura.

Su objetivo general fue establecer el perfil lector del actual estudiante de pedagogía, el valor otorgado a la lectura literaria y al rol como mediadores en su acercamiento a esta.

\section{Objetivos específicos}

-Identificar las lecturas literarias de los estudiantes de pedagogía a partir del análisis de sus historias lectoras.

-Analizar el rol de la familia, la escuela y la biblioteca como mediadores en el acercamiento a la lectura literaria.

-Indagar sobre el valor que los estudiantes de pedagogía otorgan a la lectura literaria.

En este artículo, nos centraremos en los dos últimos aspectos investigados.

\section{Universo y Muestra}

La muestra estuvo constituida por 279 estudiantes de la Facultad de Ciencias de la Educación de la Universidad Católica del Maule (Chile), pertenecientes a las carreras de Educación Parvularia (EP 2OII y 20I2), Pedagogía General Básica (PGB 2004) y de Pedagogía en Lengua Castellana y Comunicación (PLCC 2OI2, 2OI3, 2OI4,2OI5 y 2OI6). 


\begin{tabular}{|l|l|l|l|}
\hline Generaciones & Femenino $(F)$ & Masculino $(M)$ & Totales \\
\hline PLCC 2012 & 25 & 7 & $\mathbf{3 2}$ \\
\hline PLCC 2013 & 20 & 9 & $\mathbf{2 9}$ \\
\hline PLCC 2014 & 31 & 8 & $\mathbf{3 9}$ \\
\hline PLCC 2015 & 32 & 9 & $\mathbf{4 1}$ \\
\hline PLCC 2016 & 46 & 12 & $\mathbf{5 8}$ \\
\hline EP 2011 & 42 & 0 & $\mathbf{4 2}$ \\
\hline EP 2012 & 24 & 0 & $\mathbf{2 4}$ \\
\hline PGB 2004 & 13 & 1 & $\mathbf{1 4}$ \\
\hline TOTAL & 234 & 47 & $\mathbf{2 7 9}$ \\
\hline
\end{tabular}

Tabla I: Distribución según sexo

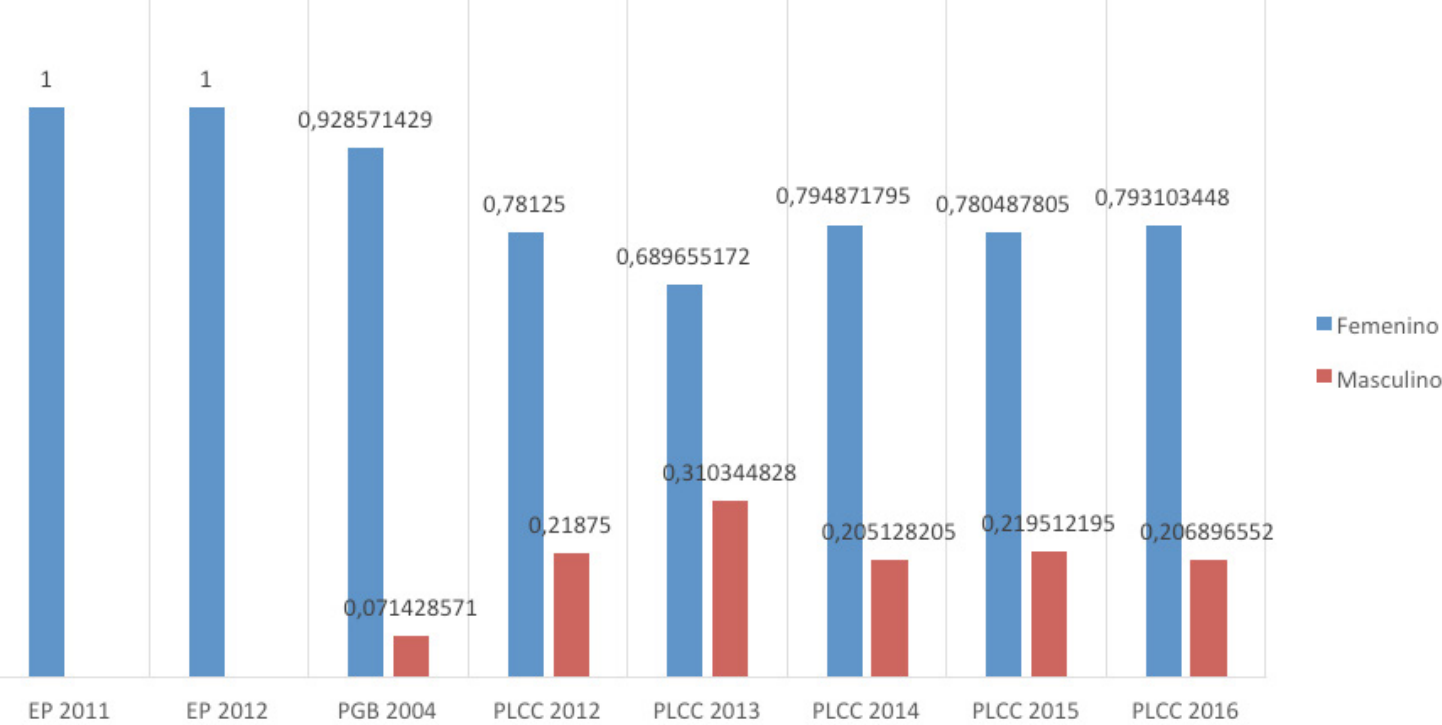

Figura I: Distribución según sexo

\section{Procedimiento de obtención de los datos}

Estos futuros profesores respondieron -en el marco de los cursos de Expresión Literaria (Educación Parvularia) y de Literatura Infantil y Juvenil (Pedagogía en Educación Básica y Pedagogía en Lengua Castellana y Comunicación) - a la siguiente consigna:

"Pidiendo ayuda a los recuerdos de infancia, recuperando imágenes, olores, sonidos y gestos, construir un texto que registre mi historia como lector/a. Preguntas que pueden ayudar:

-Mi historia con los libros: 
¿Qué cuentos o historias recuerdo de mi infancia?

¿Qué personas asocio a estas escenas?

¿Qué lugares o situaciones surgen en mis recuerdos?”

El propósito de este ejercicio era realizar una reflexión inicial que ayudara a conectar sus historias personales con su papel como futuros mediadores de la lectura literaria.

\section{Procedimiento de análisis de los datos}

Los datos se abordaron a través de un análisis mixto (estadístico descriptivo y de contenido), a partir de categorías estructurales y subcategorías determinadas por el equipo de investigadores tras varias lecturas de los textos.

Los criterios construidos respondieron a la lógica de hacer emerger las recurrencias, observadas en los discursos de los estudiantes, relacionadas con las experiencias lectoras en educación inicial, primaria, secundaria y universitaria; la frecuencia de la lectura fruitiva y el corpus de lectura mencionado; los momentos y lugares donde se realiza esa lectura; el rol de los mediadores y el valor asignado a la literatura.

\begin{tabular}{|c|c|c|}
\hline Categoría 1: Experiencia Lectora & $\begin{array}{l}\text { Categoría 2: } \\
\text { Lecturas }\end{array}$ & $\begin{array}{l}\text { Categoría 3: } \\
\text { Momentos de la lectura }\end{array}$ \\
\hline Subcategorías & Subcategorías & Subcategorías \\
\hline Experiencias de infancia & Literarias & Infancia \\
\hline Experiencias escolares (básica) & Informativas & Educación escolar (básica) \\
\hline Experiencias escolares (media) & Libros religiosos & Educación escolar (media) \\
\hline \multirow[t]{2}{*}{ Experiencia lectora actual. } & Silabario & Etapa universitaria \\
\hline & Otros & \\
\hline $\begin{array}{l}\text { Categoría 4: } \\
\text { Lugares para la lectura }\end{array}$ & $\begin{array}{l}\text { Categoría 5: } \\
\text { Rol de los mediadores }\end{array}$ & $\begin{array}{l}\text { Categoría 6: } \\
\text { Valor de la literatura }\end{array}$ \\
\hline Subcategorías & Subcategorías & Subcategorías \\
\hline Casa & Madre & Creencias \\
\hline Escuela & Padre & Representaciones \\
\hline Entorno natural & Otros familiares & Metáforas \\
\hline Otros & Escuela & \\
\hline
\end{tabular}

Tabla 2: Categorías y subcategorías 


\section{Resultados}

Los resultados que se presentarán a continuación corresponden al registro y análisis de frecuencia de las categorías 3, 4 y 5, ordenadas por carreras y generaciones. Los totales exceden el Ioo\% ya que cada sujeto participante podía realizar más de una mención en cada categoría. Las categorías i y 6, que se abordaron exclusivamente con un análisis de contenido, se consignarán más adelante. Se excluye en esta publicación la categoría 2 pues, por su nivel de detalle, se convertirá en materia de otro artículo.

\begin{tabular}{|l|c|c|c|c|}
\hline Generaciones & $\begin{array}{c}\text { Lecturas } \\
\text { de infancia }\end{array}$ & $\begin{array}{c}\text { Lecturas } \\
\text { escolaridad } \\
\text { básica }\end{array}$ & $\begin{array}{c}\text { Lecturas } \\
\text { escolaridad } \\
\text { media }\end{array}$ & $\begin{array}{c}\text { Lecturas } \\
\text { actuales }\end{array}$ \\
\hline PLCC 2012 & $66 \%$ & $78 \%$ & $28 \%$ & $6 \%$ \\
\hline PLCC 2013 & $76 \%$ & $48 \%$ & $31 \%$ & $17 \%$ \\
\hline PLCC 2014 & $82 \%$ & $95 \%$ & $46 \%$ & $28 \%$ \\
\hline PLCC 2015 & $46 \%$ & $71 \%$ & $46 \%$ & $12 \%$ \\
\hline PLCC 2016 & $40 \%$ & $22 \%$ & $14 \%$ & $3 \%$ \\
\hline EP 2011 & $21 \%$ & $60 \%$ & $10 \%$ & $5 \%$ \\
\hline EP 2012 & $75 \%$ & $38 \%$ & $13 \%$ & $0 \%$ \\
\hline PGB 2004 & $64 \%$ & $50 \%$ & $7 \%$ & $0 \%$ \\
\hline TOTALES & $\mathbf{5 9 \%}$ & $\mathbf{5 8 \%}$ & $\mathbf{2 4 \%}$ & $\mathbf{9 \%}$ \\
\hline
\end{tabular}

\section{Tabla3: Categoría 3: Momentos de la lectura}

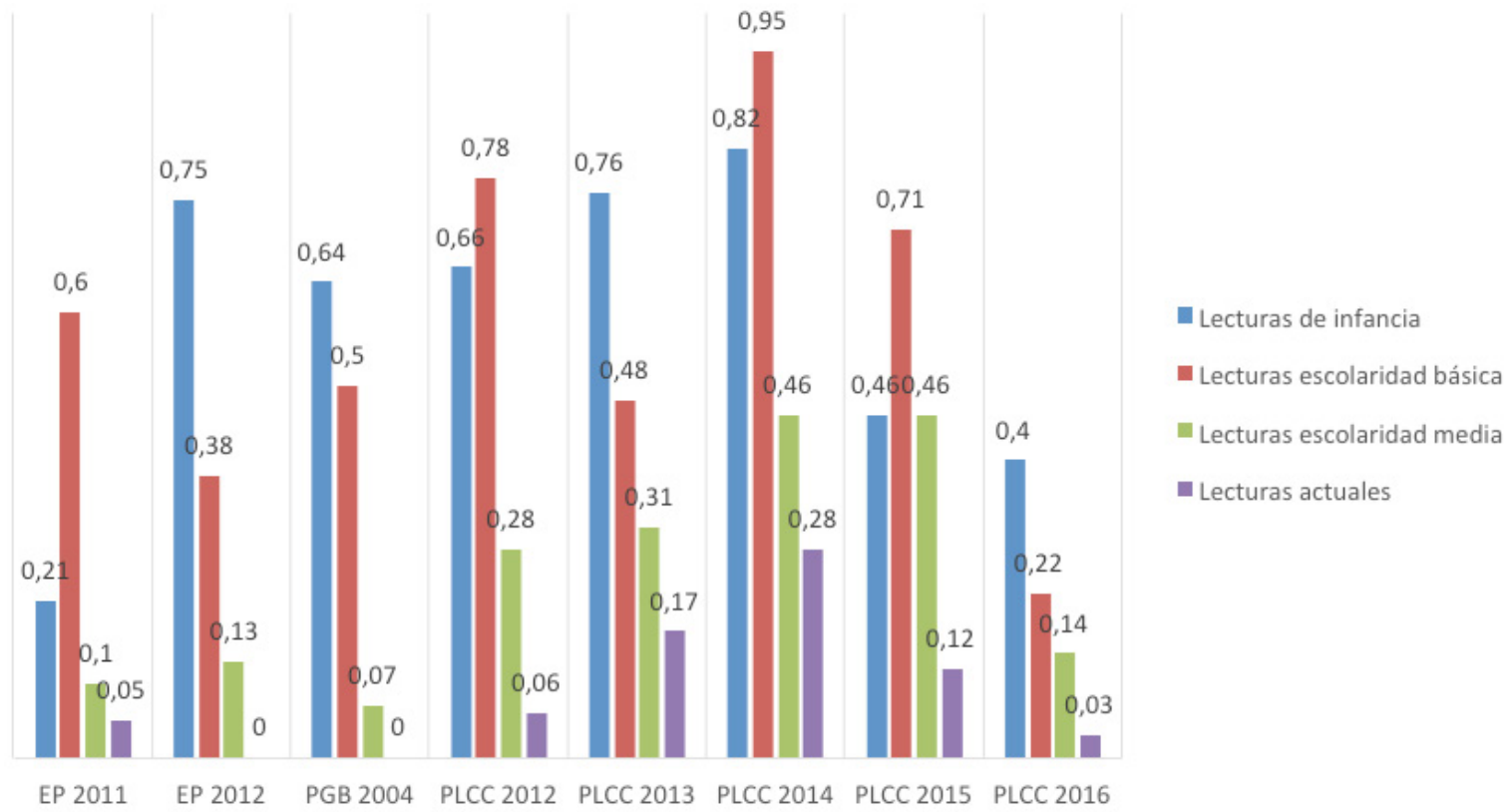

Figura 2: Momentos de la lectura 
La categoría Momentos de la lectura se definió como la identificación de los espacios temporales en que aconteció la experiencia lectora. El sistema educativo chileno consta de 8 niveles de Educación Básica (Primaria) y 4 niveles de Educación Media (Secundaria), que conforman el total de los años de escolaridad obligatoria. De acuerdo a la recurrencia de las situaciones mencionadas se determinó establecer tres momentos: infancia -entendiendo por ella el periodo previo a la escolarización-, educación escolar y finalmente las lecturas realizadas desde su incorporación a la universidad (entre dos y tres años de permanencia en esta).

Como se observa en la figura 2, los Momentos de la lectura se concentran en torno a las experiencias en el hogar antes de ingresar a la escuela básica. Tal como se advierte en la tabla 3 , la diferencia con este segundo momento es pequeña (ı\%), lo que puede deberse a que la escolarización implica el logro de la lectoescritura, que abre infinitas posibilidades lectoras.

En la enseñanza media se constata un descenso considerable en cuanto a su mención como momento de lectura (promedio 24\%) en todas las generaciones de estudiantes, a pesar del llamado plan complementario de lectura, correspondiente a un listado anual de seis u ocho libros que los estudiantes deben leer obligatoriamente para una evaluación mensual.

Como señalan Munita y Pérez (20I3), "el hecho institucionalizado, y convertido en rutina, de leer en forma obligada un libro para luego contestar una prueba sobre lo leído" (p.I8o), genera desmotivación por la lectura, situación que ha enfrentado la educación literaria en la escuela chilena. Sin embargo, no se puede desconocer que para algunos alumnos, este plan es el único contacto con el discurso literario.

Los títulos de este plan complementario de lectura son propuestos por las bases curriculares para los distintos niveles educativos, pero cada establecimiento educacional tiene la libertad de escoger otros (Ministerio de Educación (Mineduc), 20I6).

La sugerencia de las obras se relaciona con los objetivos del eje de lectura establecidos para cada curso y con los siguientes criterios de selección: trascendencia en el tiempo, ampliación de la cultura y visiones de mundo para entender la diversidad, pertinencia a la edad; a ellos se añaden que las obras seleccionadas inviten a la reflexión sobre sí mismos(as) y sobre vivencias comunes a todo ser humano, y que den pie a discusiones y comentarios escritos interesantes.

Respecto de las lecturas realizadas durante el periodo de sus estudios universitarios, presentan un escaso reconocimiento, solo con un $9 \%$ en promedio. Se destaca PLCG (2OI4) con un 28\% que contrasta con la ausencia de títulos en EP (2OI2) y PGB (2004). 


\begin{tabular}{|l|c|c|c|c|}
\hline \multicolumn{1}{|c|}{ Generaciones } & Casa & Escuela & $\begin{array}{c}\text { Entorno } \\
\text { Natural }\end{array}$ & Otros \\
\hline PLCC 2012 & $38 \%$ & $0 \%$ & $13 \%$ & $6 \%$ \\
\hline PLCC 2013 & $100 \%$ & $34 \%$ & $3 \%$ & $3 \%$ \\
\hline PLCC 2014 & $8 \%$ & $0 \%$ & $3 \%$ & $3 \%$ \\
\hline PLCC 2015 & $15 \%$ & $0 \%$ & $2 \%$ & $10 \%$ \\
\hline PLCC 2016 & $41 \%$ & $5 \%$ & $12 \%$ & $7 \%$ \\
\hline EP 2011 & $43 \%$ & $24 \%$ & $2 \%$ & $2 \%$ \\
\hline EP 2012 & $63 \%$ & $33 \%$ & $4 \%$ & $4 \%$ \\
\hline PGB 2004 & $93 \%$ & $21 \%$ & $0 \%$ & $0 \%$ \\
\hline TOTALES & $\mathbf{5 0 \%}$ & $\mathbf{1 5 \%}$ & $\mathbf{5 \%}$ & $\mathbf{5 \%}$ \\
\hline
\end{tabular}

Tabla 4: Categoría 4: Lugares para la lectura

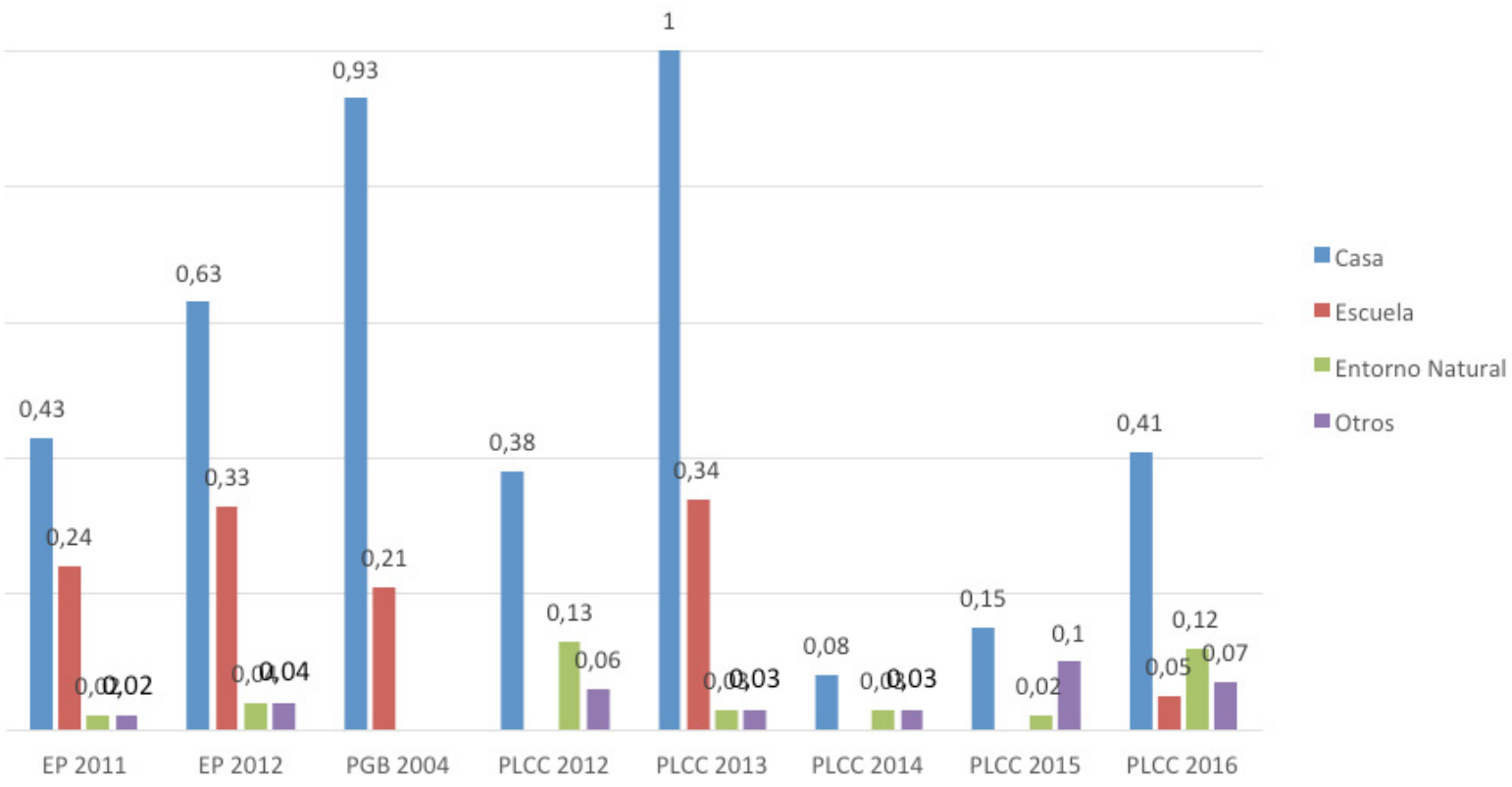

Figura 3: Lugares para la lectura 
La categoría Lugares para la lectura alude a los espacios físicos donde esta se realiza. Se observa que, en todas las generaciones de estudiantes, la casa es el lugar mencionado preferentemente, ya sea que se encuentre ubicada en la ciudad, en el campo o donde se descansa en vacaciones. Dentro de la casa, se destaca el dormitorio, donde los niños escuchaban a sus padres o abuelos narrarles historias familiares y cuentos maravillosos.

En segundo lugar, aparece la escuela, incluyéndose en ella todos los niveles de educación. Sorprende que en tres generaciones (PLCG 2012 ,20I4 y 2OI5) no exista ningún reconocimiento explícito de esta institución.

La lectura en entornos naturales y en espacios distintos a los anteriores es mencionada con escasas preferencias. Los lugares al aire libre se concentran en torno a la lluvia y los árboles, generalmente en tiempos de vacaciones invernales o de verano. Los espacios restantes que se recogen en el análisis son trayectos de viajes y la biblioteca, entre otros.

\begin{tabular}{|l|c|c|c|c|}
\hline \multicolumn{1}{|c|}{ Generaciones } & Madre & Padre & $\begin{array}{c}\text { Otros } \\
\text { familiares }\end{array}$ & Escuela \\
\hline PLCC 2012 & $34 \%$ & $13 \%$ & $28 \%$ & $0 \%$ \\
\hline PLCC 2013 & $41 \%$ & $28 \%$ & $21 \%$ & $34 \%$ \\
\hline PLCC 2014 & $49 \%$ & $26 \%$ & $21 \%$ & $28 \%$ \\
\hline PLCC 2015 & $39 \%$ & $10 \%$ & $32 \%$ & $20 \%$ \\
\hline PLCC 2016 & $60 \%$ & $22 \%$ & $34 \%$ & $7 \%$ \\
\hline EP 2011 & $45 \%$ & $26 \%$ & $10 \%$ & $21 \%$ \\
\hline EP 2012 & $96 \%$ & $38 \%$ & $33 \%$ & $17 \%$ \\
\hline PGB 2004 & $57 \%$ & $21 \%$ & $21 \%$ & $14 \%$ \\
\hline TOTALES & $\mathbf{5 3 \%}$ & $\mathbf{2 3 \%}$ & $\mathbf{2 5 \%}$ & $\mathbf{1 8 \%}$ \\
\hline
\end{tabular}

Tabla 5: Categoría 5: Rol de los mediadores 


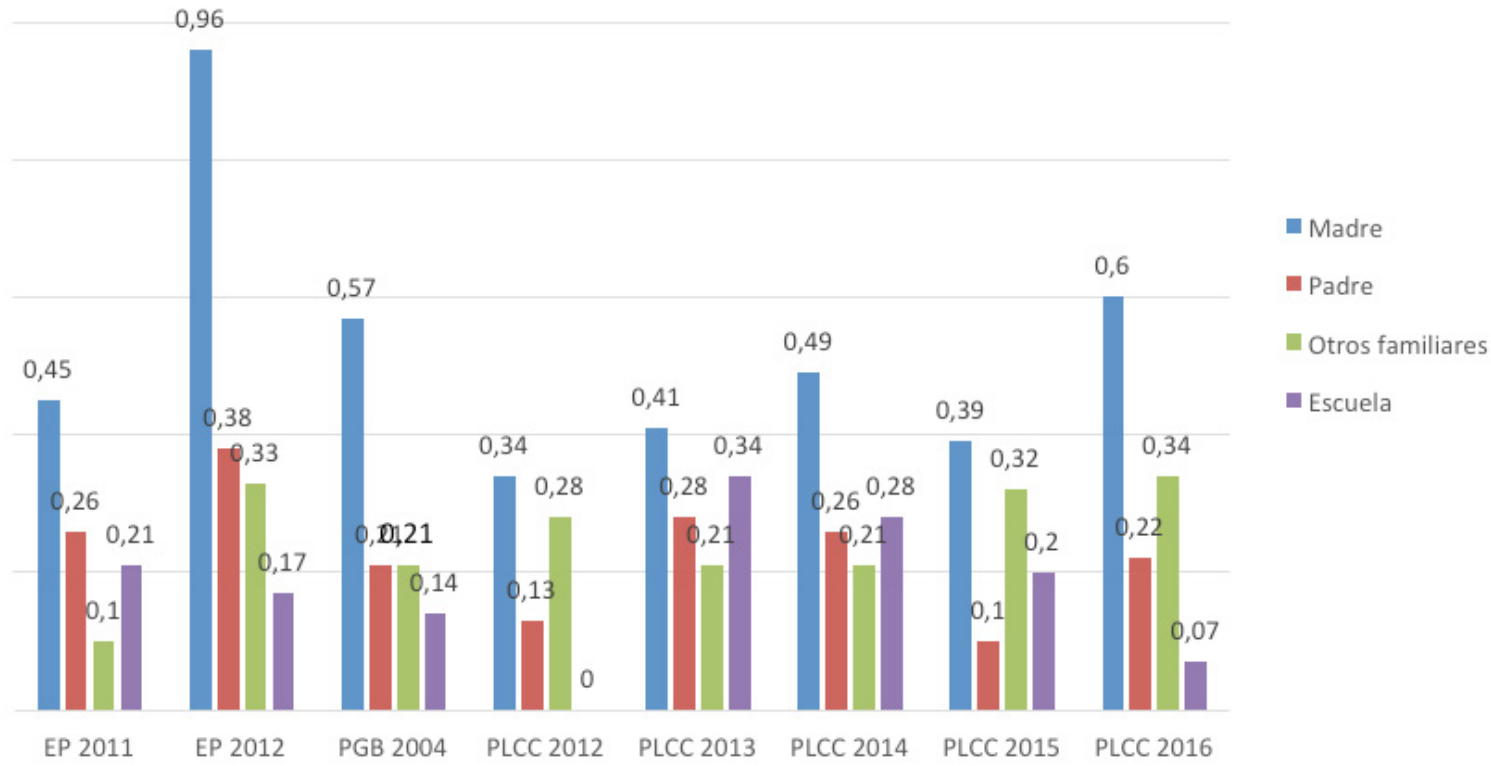

\section{Figura 5: Rol de los mediadores}

En cuanto al rol de los mediadores en el acercamiento a la lectura literaria, en todas las generaciones es la figura amorosa de la madre la que más se recuerda (promedio $53 \%$ ), evocando su voz y su olor cuando narraba cuentos o entonaba canciones para sus hijos. Se destaca EP 20I2, generación en que el 96\% de los estudiantes mencionó a esta figura.

Los siguientes mediadores identificados corresponden a otros familiares (abuelos, tíos y primos), quienes proveyeron de compañía y libros a los niños cuando sus progenitores estaban fuera de casa debido a razones laborales o cuando no contaban con herramientas adecuadas para la tarea.

A continuación, con una diferencia de $2 \%$ en el promedio, se nombra al padre y, finalmente, a la escuela (promedio I8\%), a pesar de haber sido mencionada en segunda instancia cuando se consultó sobre lugares para la lectura. Su escaso reconocimiento asociado a la mediación -nulo en el caso de PLCC 2OI2-se explicaría por la asociación entre lectura escolar y obligatoriedad.

Las siguientes categorías, correspondientes a la i y a la 6 , se abordarán desde un enfoque cualitativo con el propósito de comprender algunos aspectos de la realidad a través de una aproximación interpretativa.

\section{Categoría 1.- Experiencia lectora: infancia, escolar básica, media y actual.}

En el caso de la época infantil, se aprecia que la experiencia se sitúa en el ámbito familiar e inevitablemente se asocia a aspectos emocionales. Dicho proceso conlleva la 
creación de lazos afectivos, los cuales se construyen al ritmo de las canciones de cuna antes de dormir, siguiendo una especie de rito nocturno. Es en esta etapa cuando se rememora especialmente a la madre, quien mece al hijo mientras le canta rondas o le recita poemas, momento valioso que perdura en el tiempo. Es el periodo de escucha de mamás y abuelas, quienes rescatan la tradición oral de contar historias familiares o leyendas de la zona:

Siempre recuerdo esas instancias de lectura al anochecer, en la cama junto a mi madre y mi hermano, abrigada por el calor de nuestra madre, iluminada por la luz de la lámpara del velador, eran fascinantes esos momentos, no hallaba la hora de que llegara la noche para escuchar las fantásticas historias narradas por la dulce voz de mi madre (EP2OI2-6I).

Otro aspecto importante que se evidencia en la etapa infantil son los diversos intentos realizados por los estudiantes para aprender a leer, recibiendo el apoyo incondicional de su familia. Dicho aprendizaje es aprovechado por los padres para formar en valores, mientras se reflexiona sobre el accionar de los personajes de cuentos.

El acercamiento inicial a la lectura es placentero, se realiza desde la familia y, aunque con el paso del tiempo los gustos literarios tomen caminos distintos, la lectura deviene en instancia de unión familiar, en ocasiones, en torno al fogón, como lo hacían nuestros antepasados, para escuchar la voz de los ancianos.

Surge también, en esta etapa, un nuevo formato que fomenta el acercamiento de los niños a la lectura: los audiolibros que se encuentran en el comercio y son acompañados con los libros impresos o difundidos en la radio. A pesar de la novedad anterior, la imagen es fundamental en la formación del gusto por la lectura, pues a partir de las ilustraciones, los niños van construyendo la historia, lo que luego se complementa al colorear los dibujos que acompañan los cuentos infantiles.

Cuando el niño comienza su escolaridad básica, se produce un gran cambio. En primer lugar, el aprender a leer se relaciona con la persona que participó de dicho proceso, en este caso algún profesor. Este aprendizaje se convierte en un gran logro que se nutre con el reconocimiento y orgullo de los padres. Ahora ya no se escucha leer, sino que se lee para otros.

La experiencia lectora en este periodo se transforma por completo: "desde ese momento mi interés por los libros cambió, me gustaba abrirlos, saber lo que decían, olerlos, mirar sus dibujos, pasar las páginas, leérselos a los demás” (LCG 20I6-F-I46).

Surge la autonomía, la posibilidad de elegir qué leer. El libro se convierte en un regalo que se comparte entre amigos, hermanos o primos menores.

Destaca la recurrente mención del "Silabario", tradicional manual chileno para la enseñanza de la lectoescritura, que se transforma en herencia familiar recibida de los hermanos mayores y se delega para los menores. Esta predilección es reforzada con el recuerdo sensorial que logra evocar este texto: 
Además me gustaba frotar las hojas de mi antiguo silabario, la sensación que se genera en la yema de los dedos me relajaba, al igual que el olor de esas antiguas hojas, hasta hoy cuando leo un libro con este tipo de hoja le tomo el olor y las froto (PLCC- 2OI2-F-2I).

En segundo lugar, en esta etapa se gesta una representación que marcará la experiencia de la mayoría de los sujetos que conforman la muestra. Lamentablemente, se relaciona la lectura con la prescripción y la evaluación, transformando la inicial experiencia placentera en algo desagradable y aburrido. Las lecturas obligatorias hacen desaparecer "como por arte de magia" la emoción que provocaba en los primeros años: "mis deseos de descubrimiento se fueron opacando, el sentir que la lectura era una imposición y el saber que había un plazo y unos parámetros establecidos en la forma de leer, terminaron por derribar mi interés" (PLCC 20I6-F-I69).

Ya no se lee para pasar un buen rato ni para compartir en familia, sino que principalmente se realiza como respuesta a las exigencias del colegio y, por ende, la compra de libros también se convierte en una demanda para los padres. Se pierde el derecho a elegir qué y cuándo leer. Sin embargo, en algunos casos la escuela se convierte en el único mediador que el alumno conoce. Entonces, aunque el método no sea el más adecuado, se agradece el acercamiento a la lectura.

Lo anterior se relaciona con que en el ámbito escolar existe una escasa preocupación por crear un ambiente para la lectura (Chambers, 2007a) y propiciar su fomento, a pesar de que constituya una de sus responsabilidades. Ello coincide con las escasas menciones a la escuela como espacio para la lectura. En este mismo sentido, el espacio educativo estaría desoyendo una de las indicaciones de las bases curriculares (Mineduc, 2OI6):

Es de la mayor importancia que los textos escogidos por el o la docente sean interpretados y comentados en clases. Esto significa que, en la medida de lo posible, las obras se lean en conjunto con la profesora o el profesor, ya sea íntegramente o una parte, dependiendo del tiempo, de la extensión y de la dificultad de la obra, y luego se analicen e interpreten en clases, a partir de las contribuciones de las y los estudiantes (p.79).

Actualmente, desde el campo de la didáctica de la literatura, se reconoce la centralidad de la discusión o conversación literaria (Munita, 20I8; Chambers, 20O7b) como la estrategia más recomendable para la mediación. Ello coincide con uno los criterios que deberían orientar la selección de los títulos ("den pie a discusiones y comentarios escritos interesantes").

Aquí se releva el rol que debe desempeñar el profesor quien deberá “ayudar a los y las estudiantes a penetrar en el significado, trabajando especialmente el vocabulario, entregando una contextualización, relacionando con otros textos y otras experiencias y fomentando la aplicación de las estrategias de lectura en los casos que sea pertinente"(p.79). 
Solo de esta manera es posible estimular el desarrollo del pensamiento crítico y la apreciación de la literatura con fines de goce estético.

Por último, es en este periodo cuando, para algunos jóvenes, leer literatura se convierte en una necesidad, pues los libros se transforman en una vía de escape a la realidad, que motiva a refugiarse en los clásicos infantiles o en la literatura que los aparta de vivencias dolorosas y los seduce con entretenidas peripecias: "cuando estaba en mi etapa adolescente, la lectura se transformó en un universo que me permitía vivir aventuras, romances, viajar entre espacios y tiempo, conocer nuevos lugares y experimentar emociones (PLCG 20I6-F-I58).

En cuanto a la siguiente etapa de esta categoría, donde se recuerda la experiencia lectora desde lo vivido en la educación secundaria, se aprecian algunos aspectos relevantes, siendo el más recurrente la lectura por obligación. Esta práctica se instrumentaliza y se convierte en el medio acostumbrado, en las clases de Lenguaje, para obtener calificaciones a partir de la lectura mensual obligatoria. Ahora, los textos son más complejos y más largos; en consecuencia, el aburrimiento también se extiende.

Algunos participantes del estudio recuerdan la experiencia lectora con indiferencia: "y como mencioné anteriormente ya de grande no leía los libros para las notas en el liceo, me aburrían y no me gustaban. Ya ahora he tenido que adquirir ese hábito, no es fácil [...] y no creo haberme perdido nada durante la enseñanza media.” (EP2OII-23).

Felizmente, otros lectores la rescatan a pesar del desinterés inicial: "cuando me di cuenta que la literatura era mucho más que los aburridos libros que daba la profesora, que había poesía en textos desconocidos, me arrepentí profundamente por todos los años en que pasé indiferente frente a estanterías y ferias de libros" (PLCC2OI2-F-32).

En la adolescencia, se inicia la recomendación de títulos y autores entre los amigos que comparten el mismo gusto lector. En este periodo, el joven elige voluntariamente qué leer fuera del aula escolar, y lo guía el grado de complicidad e identificación que logra con los textos y sus temáticas: “me devoré sus hojas: lloré, reí, sentí pena y dolor [...] Era mi teleserie en libro, vivida como si fuera mi propia historia cada vez que cambiaba de página" (EP 2OII-33).

Junto a este hecho, se comienza con la lectura en formato virtual, lo que facilita la adquisición del libro y, con ello, el ahorro familiar del costo asociado. Aunque este medio les permite una adquisición rápida e inmediata del texto, no se pierde el gusto por el texto impreso, el que se puede hojear y sentir: “aquel libro olía a café de trigo... olía a sueños... a imaginación... a superación. Olía a la vida que llevé por siete años” (LCC 2OI3- F- 53).

Para algunos alumnos, la lectura ya forma parte de sus vidas y logran transitar desde la lectura comercial a la clásica, sin dificultades. Estos sujetos leen por entretención todo lo que llega a sus manos, sin discriminar formatos, y "devoran" revistas, comics, novelas, fanfiction, etc. De esta manera, y guiados solo por el disfrute, el gusto por la lectura se fortalece con el paso del tiempo y no logra afectarse con las imposiciones escolares. En ocasiones, dicha afición es motivada por algún profesor que ha respetado la libertad de elegir. 
Finalmente, en el análisis de la etapa universitaria, los participantes del estudio señalan los pro y los contra de recibir formación literaria; unos tienen la creencia de que el conocimiento adquirido no solo les permite discriminar y ser más críticos al momento de elegir un texto, sino que también les resta el entusiasmo, el asombro e inocencia de las primeras lecturas: "ahora con veinte años, siento que cada vez es más difícil leer un libro y que este me provoque algo, porque siento que mi criterio se ha ido formando y no me entusiasman las cosas que en mi niñez sí” (LCC 2OI6-F-I47).

Sin embargo, otros consideran lo contrario pues valoran los criterios estéticos y literarios como herramientas para transformarse en mejores mediadores, tanto de sus futuros alumnos como de su propia familia. Un tercer grupo, confía en que el ingreso a una carrera de pedagogía logre fomentar el gusto por la lectura, que aún no se ha adquirido hasta ese periodo.

Hay que considerar que una vez que se despierta el placer por la lectura,este encuentro deja una huella indeleble, y que puede ir y volver en la vida, dependiendo de las experiencias que se relacionen con ella: "descubrí entonces cómo la literatura me va ayudando a entender mi realidad y no a escapar de ella, y así fue cómo el gusto y el goce por las lecturas volvió" (LCC 20I6- F-I66).

\section{Categoría 6.-. Valor de la literatura}

Esta última categoría se abordará relevando la voz de los participantes, a través del análisis de las metáforas empleadas para referirse a los libros. Estas nos permiten ahondar en las creencias y representaciones de los lectores sobre el valor de la lectura, entendiendo que las primeras pertenecen al imaginario individual y las segundas se sitúan en aquellos principios y valores compartidos (Munita, 20I6).

El aprendizaje de la lectura es considerado como el más importante, pues cambia y transforma la vida:

...lo magnífico fue salir a la calle y empezar a juntar esas letras, formar esas palabras y frases en todo lo que estaba a mi alrededor: letreros, anuncios, direcciones. Todo comenzó a tomar sentido, lo que hacían, decían y leían las personas. En pocas palabras aprendí a leer el mundo" (PLCG 20I6-M-I64).

El principal factor que convierte la experiencia lectora en algo inolvidable es que se presenta como algo único y significativo para cada lector: "fue así como me di cuenta que existe algo en los libros que no sabría bien cómo explicarlo, porque es un descubrimiento personal que llega cuando menos te lo esperas y te marca para el resto de tu vida" (EP2OII-33). A partir de esta idea, se podría considerar la posibilidad de que exista "un libro para cada lector," lo que haría realidad dicha conexión.

Es así como se pueden señalar una serie de metáforas que surgen en las narrativas de las historias de lectura recopiladas paraesta investigación: El “libro sabio" que contiene 
historias llenas de riqueza, lo que hace hablar de "libros ancianos"; el libro presentado como un "tesoro" que esconde un pirata en una isla remota, cuyo valor no se extinguirá mientras su lectura perdure y trascienda en el tiempo.

Los textos también se definen como compañeros ideales: “ese único cuento del que tengo recuerdo, se transformó en mi mejor amigo. Mi compañía en mi soledad de niño" (PLCC2OI2-M-I5). Están presentes en las distintas etapas de la vida, y se convierten en el "refugio seguro", en un salvavidas, permitiendo que el lector pueda resguardarse entre sus páginas, o en la historia que lo cautiva y, en algunos casos, lo rescata:

... fueron y son mis escudos en contra de monstruos, mis pesadillas, mis miedos, son ese compañero cuando la tristeza te ahoga y crees que la única solución es despedirte de todo, son aquel barco que por muy frágil que se ve soporta a las más crudas tormentas en el mar de nuestro interior, son ellos quienes están cuando no hay quien esté junto a mí, esperando, mientras aún sigo en pie (LCC 20I6-Fi75).

Los libros se reconocen también como "castillo de letras" o" ladrillos que levantan los muros" necesarios para protegerse.

La lectura se convierte en el "avión particular" que transporta al lector a diferentes mundos, recreando nuevas vidas y experiencias: "mis lecturas me llevaron a otras épocas, a otros continentes, con historias de espadas mágicas, caballeros valerosos" (PLCC 2OI2-F-28). El libro es un “compañero de viaje” desde la niñez, desde que se logra pronunciar las primeras sílabas, pues "saber leer... abre puertas a mundos mágicos, a aventuras que te hacen sentir libre, a mundos que ayudan a buscar la identidad, a mundos que te hacen reír como también llorar" (PLCG 2OI2- F-25).

Identificarse con las temáticas de los libros es un factor esencial en la elección y disfrute de la lectura; surge inevitablemente la "necesidad de elegir", lo que permite desarrollar la empatía a partir de las experiencias que viven los personajes, ya que "no necesitas vivir en todas las realidades para conocerlas y aprender y empatizar con ellas" (LCC2Or4-M-97). La lectura permite "vivir más de una vida”.

Los libros también generan un vínculo muy poderoso con otros que también comparten el placer de la lectura: “con mi mamá creábamos una conexión increíble cuando ella me leía, sentía que nuestros lazos se estrechaban mucho más y era una de las sensaciones que más me gustaba experimentar en nuestras noches de libros" (PLCC 2Or6-F-I53).

El primer acercamiento a la lectura y la emoción con que se recuerdan los primeros relatos, son la imagen que perdura y que logra unir generaciones y familias, venciendo el paso irremediable del tiempo: “arrastro canciones, cuentos y novelas cuyos personajes principales comparten el ADN familiar” (PLCC 20I6-F-I93). 


\section{Discusión y conclusiones}

Las narrativas de lector revisadas en el contexto de esta investigación nos entregan información sobre qué lecturas, personas, momentos y lugares han construido las biografías de un grupo de profesores en formación. En su análisis hemos atendido a los momentos iniciales de la lectura en el contexto familiar y escolar para detectar dónde radica la motivación por esta práctica y qué obstáculos la desaniman. Hemos avanzado en el tiempo, intentando construir perfiles diacrónicos, con el propósito de detectar las conexiones entre una mayor o menor inclinación hacia la lectura literaria de los sujetos del estudio y las formas de mediación de que han sido objeto.

Las conclusiones que se desprenden del recorrido anterior son coincidentes con las propuestas por las investigaciones realizadas en el contexto español. Una de las más relevantes es el valor de la lectura compartida. El acto de leer, para y con otros, es, sin duda, una experiencia generosa e inolvidable que, en los primeros años, se vive en familia. Así como se destaca el rol mediador de las figuras parentales, incluyendo a abuelos y tíos, sorprende constatar la escasa mención del profesor. La razón estaría en la obligatoriedad asociada a la lectura en este espacio.

Cuando esta se vuelve autónoma, desaparece el mediador familiar. Ello no significa que el valor de la lectura se vea disminuido pues, para los padres, esta conquista es central para el desarrollo cognitivo y cultural de sus hijos, aunque ellos no se definan actualmente como lectores, e incluso, no hayan leído o narrado cuentos a sus hijos.

Lo que desaparece en esta etapa es la relevancia del placer lector, pues los padres avalan la imposición de la lectura. En la escuela se exigen ciertos textos, pero se dedica escaso tiempo a apropiarse de ellos, por lo tanto, no se genera la afición lectora. Tal como distingue Arizaleta (2009) existe una diferencia entre prescribir o mandar a leer textos determinados, y realizar una mediación, entendida esta como invitación a la lectura de ciertos textos seleccionados por el adulto.

Esta situación se hace más evidente en el paso a la enseñanza secundaria, tal como lo relatan Dueñas et al. (2OI4). En esta etapa, serán los amigos quienes acercarán los nuevos títulos a los lectores adolescentes (Contreras y Prats, 2OI5), no pocas veces seducidos por los mecanismos de enganche propios de la literatura comercial (Lluch, 2006; 2007).

A pesar del planteamiento anterior, en ocasiones, la imposición de los libros se convertirá en el único camino de llegada a la literatura para quien tiene difícil acceso a este bien o no ha construido sus propios criterios de selección (Contreras y Prats, 20I5). Se destaca en la pesquisa que, no pocas veces, es el profesor quien motiva incluso la elección de una carrera vinculada con las letras. Particularmente ello es mencionado por los estudiantes de Pedagogía en Lengua Castellana.

Se constata la ausencia de la biblioteca como tercer agente participante en la tríada de la mediación, junto a la familia y la escuela. Son pocos los recuerdos de bibliotecarios escolares o municipales que recomiendan y estimulan a vivir la aventura lectora. La causa la podríamos encontrar en la, en ocasiones, insalvable distancia entre las funciones que corresponden a la biblioteca escolar y su cumplimiento (Mekis y Andwanter,20I9). 
Otra de las conclusiones a la que hemos llegado es la importancia de lo sensorial en la generación del placer lector. Muchas veces, se aludirá en los testimonios al gusto de oler las hojas gastadas y oírlas movidas por el viento, de tocar su textura y mirar con profusión las ilustraciones. Nos preguntamos si no será esta una de las razones que asegurarán la pervivencia del libro impreso.

Finalmente, quisiéramos destacar una de las principales motivaciones que sostienen el gusto por la lectura: la identificación con los personajes de las historias. Esta razón, que cobra especial fuerza en la adolescencia, puede correr el peligro de emplear la literatura con propósitos ideologizadores, toda vez que reproduzca estereotipos y patrones que se siguen sin cuestionamiento (Lluch, 2006; 2007).

La cara positiva del mismo mecanismo de enganche es la posibilidad de vivir experiencias vicarias: "Sentí empatía por Tom, el esclavo negro, me sentía como él porque mi piel era más oscura que la de mis compañeros" (PLCG 2OI2-25- F).

Dar la posibilidad de "vivir más de una vida" a través de la literatura, "la representación de una historia que quizás pudo suceder", en palabras de los estudiantes consultados, es un compromiso que involucra no solo a la familia, la escuela y la biblioteca sino a todo el país. Especialmente cuando se garantiza la lectura como un derecho social para todos los habitantes. 


\section{Referencias bibliográficas}

-Arizaleta, L. (2009). Prescripción y mediación. Dos actitudes ante la educación literaria. CLIJ, año 22, 232, I9-23.

- Cassany, D. (2012). En línea: leery escribir en la red. Barcelona: Anagrama

- Cerrillo, P. y Senís, J. (2005). Nuevos tiempos, ¿nuevos lectores? Ocnos, I, I9-33. doi: http://dx.doi.org/IO.I8239/ocnos_2OO5.OI.O2

- Consejo Nacional de la Cultura y las Artes (2015). Plan Nacional de la Lectura 2015-2020.

- Consejo Nacional de la Cultura y las Artes (2018). Plan de la Lectura. Región del Maule $2017-2022$.

- Colomer, T. y Munita, F. (20I3). La experiencia lectora de los alumnos de Magisterio: nuevos desafíos para la formación docente. Lenguaje y textos, 38, 37-45. Obtenido el I 4 de septiembre de 2018 desde http://www.sedll.org/sites/default/files/journal/la_experiencia_lectora_de_los_alumnos_de_magisterio._colomer_t.pdf

- Contreras, E. y Prats, M. (2015). ¿La educación literaria de los futuros maestros es suficiente para ejercer de mediadores de las nuevas generaciones? Vía Atlántica, 28,29-44. Obtenido el I de octubre de 2018 desde https://www.revistas.usp.br/viaatlantica/article/ view/98568/IO7128

- Chambers, A. (2007 a). El ambiente de la lectura. México: FCE

- Chambers, A. (2007 b). Dime. México: FCE

- Dueñas, J.D., Tabernero, R., Calvo, V. \& Consejo, E. (2OI4). La lectura literaria ante nuevos retos: canon y mediación en la trayectoria lectora de futuros profesores. Ocnos, II, 2 I-43. doi:http://dx.doi.org/IO.I8239/ocnos_2OI4.II.O2

- Granado, C.\& Puig, M. (2OI4). La identidad lectora de los maestros en formación como componente de su identidad docente. Un estudio de sus autobiografías como lectores. $O c$ nos,I3, 43-63.doi:http://dx.doi.org/IO.I8239/ocnos_2OI5.I3.O3

- Leibrandt, I. (20IO). Las herramientas electrónicas en la didáctica de la literatura. Revista Digital Universitaria, Vol.II, ${ }^{\circ} 9,3^{-}$I5

- Lluch, G. (2006). Mecanismos de adicción en la literatura juvenil comercial. Biblioteca Virtual Universal, I-I7. Obtenido el I de julio de 2018 desde http://www.biblioteca.org.ar/ libros/I34633.pdf 
- Lluch, G. (20O7). Literatura infantil y juvenil y otras narrativas periféricas. En P. Cerrillo y C. Cañamares (Coords. Literatura infantil: nuevas lecturas y nuevos lectores (pp.193-2I2). España: Ediciones de Castilla La Mancha

- Lluch, G. (2OI4). Jóvenes y adolescentes hablan de lectura en la red. Ocnos,II,7-20. Obtenido el I de octubre de 2019 desde http://www.revista.uclm.es/index.php/ocnos/article/ view/44I

- Mekis, C. y Andwanter,C. (20I9). Bibliotecas escolares para el siglo XXI. Desarrollo de comunidades de lectura. Madrid: Narcea

- Ministerio de Educación (20I6). Bases curriculares Lengua y Literatura $7^{\circ}$ básico a $2{ }^{\circ}$ medio. Santiago de Chile

- Munita, F. (2OI3). Creencias y saberes de futuros maestros (lectores y no lectores) en torno a la educación literaria. Ocnos, 9, 69-87. doi: http://dx.doi.org/IO.I8239/ocnos_2OI3.09.04

- Munita, F. y Pérez, M. (2OI3).”Controlar" las Lecturas Literarias: Un estudio de casos sobre la Evaluación en el Plan de Lectura Complementaria de Educación Básica. Estudios Pedagógicos XXXIX,I, I79-198

- Munita, F. (20I6). Prácticas didácticas, creencias y hábitos lectores del profesor en una escuela exitosa en la promoción lectora. Ocnos,I5 (2), 77-97. doi: http://dx.doi.org/IO.I8239/ ocnos_2OI6.I5.2.II4O

- Munita, F. (20I8). El sujeto lector didáctico: “lectores que enseñan y profesores que leen”. Alabe, I7,I-I9 .doi:http://dx.doi.org/ IO.I5645/Alabe2OI8.I7.2

- Munita,F. (2018.) De lo que sucedió a Catalina, y del donoso y grande escrutinio al mediador de lectura. Revista Tantágora. Obtenido el I5 de julio de 2018 desde revista@tantagora. net

- Tabernero, R. (2013). El booktrailer en la promoción del relato. Quaderns de Filología. Estudis literaris. Vol XVIII,2II-222 